\title{
Variable Admittance Control for Climbing Stairs in Human-Powered Exoskeleton Systems
}

\author{
Ahmed AIA ${ }^{1 *}$, Cheng $\mathrm{H}^{1}$, Lin $\mathrm{X}^{1}$, Omer $\mathrm{M}^{2}$ and Atieno $\mathrm{JM}^{3}$ \\ ${ }^{1}$ Center for Robotics, School of Automation, University of Electronic Science and Technology of China, 611731 Chengdu, China \\ ${ }^{2}$ School of Automation, University of Electronic Science and Technology of China \\ ${ }^{3}$ School of Electronic Engineering, University of Electronic Science and Technology of China, 611731 Chengdu, China
}

\begin{abstract}
Online gait control in human-powered exoskeleton systems is still rich research field and represents a step towards fully autonomous, safe and intelligent navigation. Admittance Controller performs well on flat terrain walking in human-powered exoskeleton systems for acceleration and slowdown. We are the first who proposed Variable Admittance Controller (VAC) for smooth stair climbing control in Human-Powered Exoskeleton Systems. Trajectory correction technique transforms the interaction forces exerted on the exoskeleton from the pilot to appropriate intended joint flexion angles through dynamic viscoelastic models. We demonstrate the proposed control strategy on one degree-of-freedom (1-DOF) platform first, and then extend to the Human power Augmentation Lower Exoskeleton (HUALEX). The experimental results show that the proposed gait transition control strategy can minimize the interaction dynamics with less interaction force between the pilot and the exoskeleton compared to the traditional admittance controller. Compared to Ordinary Admittance Controller, the proposed VAC significantly improve the normalized Mean Squared Error (nMSE) of trajectory tracking from $2.751^{\circ}$ to $1.105^{\circ}$
\end{abstract}

Keywords: Admittance control; Variable admittance; Gait transition; Interaction force; Coupled human-exoskeleton System

\section{Introduction}

A lot of researches on Human-Powered Exoskeleton Systems has been focused on flat terrain walking while stair ascent and vice versa is frequent process on daily life activities. Human-exoskeleton systems designed for constraining human movements to allow people to operate more easily or more efficiently in a variety of situations, required consideration of economical issues. Here we mean these systems must have simple and available control methods so can have further developments. If we take BLEEX control methodology Sensitivity Amplification Controller (SAC), efficient control way to shadow human motion but so expensive and resource consumer (hardware and software) [1].

During the navigation of coupled human-exoskeleton systems stairs ascending or descending is frequent process especially when applied for indoors mission. Several studies were performed to investigate normal human stair ascent and descent [2], investigations of biomechanics and motor coordination in human lower extremity during ascent and descent walking at different inclinations [3-5]. Other investigations such as staircase climbing of patients with knee and hip $[5,6]$.

The physical Human-Robot Interaction pHRI as a result for human mind intention is a cooperative activities between separately human and exoskeleton [7] or close physical interaction [8] are big challenge, but in coupled human-robot system is more challenge because additional issues must considered because coupled human-robot system or exoskeleton robots act directly on the human body. The pressuresensitive devices are widely used for gait analysis [9], a few researches are conducted to use such technique for measure gait variability [10] and for gait control $[11,12]$. Around thigh force-sensitive device is used for wearer intention estimation it can autonomously achieve smooth transition between different gates. Around thigh wearable force sensor device is designed to act as a sensory feedback tool to monitor the human exoskeleton interaction as well as to use it for gait transition control. The use of this interaction force as a feedback signals for our control system is efficient way for good results. The use of forcesensitive device in the end-effector of the intended joints can Figure out human intentions in clear and accurate way. For autonomous and smooth transition between different gaits we apply calibrated and efficient signal collector sensors. The wearable force sensor devices comparing to fixed (eneorized treadmills) are the best solution for on-line applications. The extracted feedback signals can monitor the human exoskeleton interaction as well as control the gait transitions.

An admittance controller also called position based impedance controller, uses the end-effector interaction force feedback to estimate the appropriate joint position. The desired joint position and position feedback are used to estimate the appropriate actuators inputs [13]. Since Neville Hogan first introduced impedance controllers [14], they have become well established specially in robotics and coupled humanexoskeleton system. The application of an impedance control strategy to coupled human-exoskeleton system will allow variable deviation from a given joint trajectory to intended maneuvers according to pilot. The Lokomat is a bilateral robotic orthosis that is used in conjunction with a body weight support system to control patient leg movements in the sagittal plane. The Lokomat hip and knee joints are actuated by linear drives, which are integrated in an exoskeletal structure. Admittance control extensively utilized in rehabilitation robotics but also was applied to upper limb power augmentation exoskeletons in

*Corresponding author: Abusabah IA Ahmed, Center for Robotics, School of Automation, University of Electronic Science and Technology of China, Chengdu, China. Tel: 8615928716860; E-mail: abusabah22@hotmail.com

Received October 06, 2016; Accepted October 26, 2016; Published October 28, 2016

Citation: Ahmed AIA, Cheng H, Lin X, Omer M, Atieno JM (2016) Variable Admittance Control for Climbing Stairs in Human-Powered Exoskeleton Systems. Adv Robot Autom 5: 157. doi: 10.4172/2168-9695.1000157

Copyright: (c) 2016 Ahmed AIA, et al. This is an open-access article distributed under the terms of the Creative Commons Attribution License, which permits unrestricted use, distribution, and reproduction in any medium, provided the original author and source are credited. 
many researches [15-19], for robot human Quadrocopter [20] and for wheel chair control [21].

We propose variable admittance controller for smooth stairs ascent to handle trajectory changes. The implemented control strategy minimizes interaction forces during stairs climbing (i.e. the exoskeleton and the pilot are in contact in variety of places).Our proposed strategy minimizes the interaction forces by translate them to desired motion and keep interaction force within accepted specified thresholds. In this work, we show how to learn admittance controller parameters online using observations stairs ascent trails. To prove the performance efficiency of proposed we show how to learn admittance controller stiffness parameters on-line using observations stairs ascent trails. To prove the performance efficiency of proposed control strategy a simulation on 1-DOF exoskeleton platform is demonstrated for normal admittance control and modified one. The result comparison shows great minimization on interaction force and in computational effort. We choose admittance controller in this work because our exoskeleton system HUALEX designed with sensitive force sensors which can provide robust feedback signal for control scheme.

The paper is organized as follows: Section 4 shows the description and integration of the human-exoskeleton system, and the ordinary admittance control performance on gait transition. We validate the efficiency of variable admittance control in Section 5 by simulation on 1-DOF platform. Section 6 shows the performance efficiency of proposed strategy on interaction force and tracking error minimization. Finally, conclusions and some perspective on future uses and further development of this technique drawn in section 7 .

\section{Human-Exoskeleton System Design}

\section{A. Dynamic models}

Totally 10-DoFs revolute joints are adopted for the HUALEX design. Among them, two-linkage revolute mechanism regarded as a 2-DOFs multilink pendulum is actuated parallel to the human thigh and shank when the exoskeleton is coupled to the pilots. The remaining DOFs are un-actuated and passively driven with in human joints movement. The appearance diagram of HUALEX is shown in Figure 1. As a wearable exoskeleton, the motion range for each DOF of HUALEX is designed according to human kinematics with some

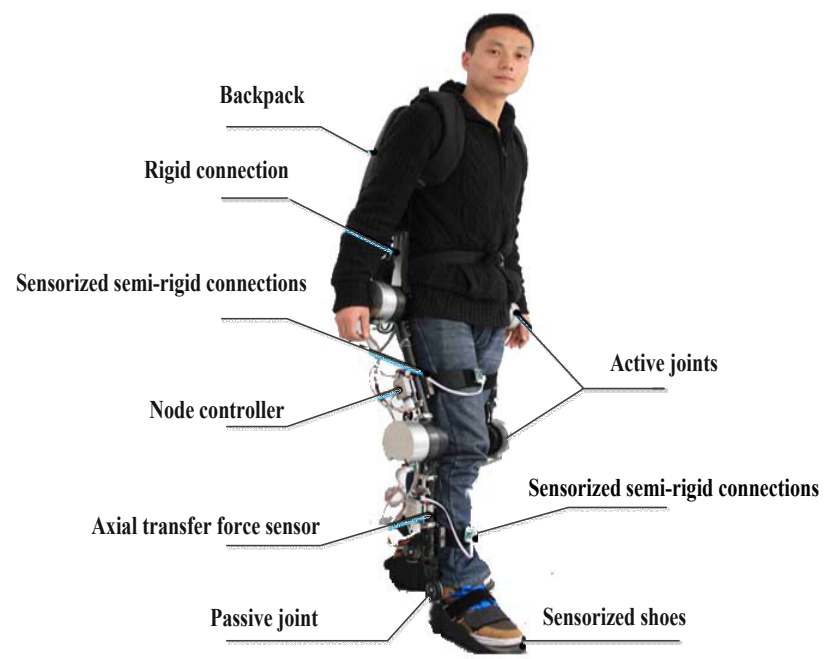

Figure 1: The prototype of HUALEX. slight differences due to flexible connections between the exoskeleton and wearer. In the sagittal plane, the designed ranges of motion at the hip, knee and ankle joints are $-45^{\circ}$ to $+45^{\circ}, 0^{\circ}$ to $-135^{\circ}$ and $-30^{\circ}$ to $+30^{\circ}$, respectively. The exoskeleton links are made with ideal design (minimum weight and inertia). The Link lengths are adjustable respect to various pilots.

A lot of researches are conducted as a series and continuous modifications for HUALEX control and performance developments. Fuzzy-based impedance regulation for control of the coupled humanexoskeleton systems [22]. The learning approach of the relationship between physical human-exoskeleton interaction and dynamic factors [23]. A modification for BLEEX SAC successfully achieved and applied for HUALEX control [24], Radial Basis Function Neural Network (RBFNN) designed to compensate for the dynamic uncertainty error and minimize the physical human-robot interaction force [25]. Force sensing technology is also an important feature in human-exoskeleton systems for monitor the interaction between pilot and exoskeleton and controls these systems. Because this sensor is additional control device for the human-robot system, it is important for the sensor to be small, lightweight, and non-invasive. Climbing stairs requires active knee extension i.e. additional torque must be applied [3]. The degree of knee extension depends on the height of stairs which is stochastic value. A higher stair will require you to bend your knees more deeply and the greater the amount of flat terrain walking knee flexion. The stairs for this work are designed with step height, and fixed tread length. The proper interaction force sensors of HUALEX make successfully investigation of admittance controller, therefore perfect angle correction. The practical measurements of joint angles, encoders on HUALEX and inclinometers on human limbs beside interaction forces between them are used to investigate the joint flexions for different stair height. Two-dimensional Interaction Force Sensors (TIFS) are developed to measure quasi-interaction force resulting from human on the exoskeleton. The information from this sensor aimed to obtain the desired change in the input walking trajectory when human intend to change gait type. It is especially useful when controlling the desired admittance between the human and HUALEX for transit from flat terrain walking to stair ascent. Since our proposed control strategy for gait transition is a model-based control strategy, the dynamic model of HUALEX project must be given.

$$
M(\theta) \ddot{\theta}+C(\theta, \dot{\theta}) \dot{\theta}+G(\theta)=\tau_{E x o}+\tau_{h}
$$

in which $\theta$ is the vector of each joint angle, $\tau_{E x o}$ and $\tau_{h}$ represent the input torques from HUALEX and human wearer, respectively. $M(\theta)$ is the inertia matrix and a function of $\theta, C(\theta, \dot{\theta})$ is the Coriolis matrix and a function of $\theta$ and $\dot{\theta}$, and $G(\theta)$ is a vector of gravitational torques. During human-exoskeleton system navigation $\tau_{h}$ is changing according to human intentions

\section{B. Admittance Control}

The admittance of the human leg shank can be given as:

$$
Y_{h}(S)=\frac{1}{Z_{h}(S)}
$$

Which characterized by inertia moment $J_{h}$, damping $B_{h}$, and stiffness $K_{h}$. The desired set of admittance parameters is required to be achieved online so that the dynamics of human-exoskeleton system interaction behaviour controlled and transferred to appropriate joint flexion correction. The interaction force on shank is measured as described in details for HUALEX with specified wearer [22]. Through inverse dynamics the end-effector measured interaction force $f_{i}$ 
transferred to joint space interaction torque $\tau_{i}$. The admittance of the coupled human-exoskeleton can be described as follows:

$$
\Delta \theta(s)=\frac{\tau_{i}(s)}{J s^{2}+B s+K}
$$

where $J, B$ and $K$ are systems inertia moment, damping and stiffness respectively. Admittance function determines the joint angular deviation through inverse kinematics. Hence the purpose of the admittance controller is to keep $\Delta \theta$ the difference between $\theta_{h}$ and $\theta_{E x o}$ as small as possible during system navigation. The resulting new reference position $\theta_{d}^{*}=\theta_{h}+\Delta \theta$ is then fed into PD controller. Experimental simulations are conducted to obtain admittance parameters. Torque or moment of force is the tendency of a force to rotate the link about the joint. Using this truth we easily can translate end effector interaction force $f_{i}$ to joint space interaction torque $\tau_{i}$ consider the distance from the interaction force point to the joint $\mathrm{l}=(0.366)$. The regulation of parameters of admittance controller in Eqn. (3) depends on human intention. The human intention resulted in interaction force which must be minimized during admittance controller by perfect shadow for human intended movements. During human-exoskeleton system navigation the variation on field terrains will lead to different intentions, in other words to variations in interaction force. As we can measure the interaction force between HUALEX and wearer we chose admittance control scheme to develop gait transitions methodology. The schematic diagram of 1-DOF platform considered in this paper is shown in Figure 2 and the admittance model is presented in Figure 3.

During human-exoskeleton system navigation the interaction force in shank strap online transformed to equivalent joint torques at knee joint through trajectory modification. Therefore, for human performance augmentation purpose, the admittance controller drives HUALEX to shadow human intended motion in the sagittal plane. Human walking process is three dimensional but the largest motions are in the sagittal plane. For calculation simplification in power augmentation human-robot systems performance analysis will take in consideration the human biomechanics in the sagittal plane. Especially for stair ascent gait the trajectories and joints variables can be obtained easily on the sagittal plane. The main goal of coupled humanexoskeleton systems control is the absolute harmony and smooth flow of human intentions acquired through different sensors and sensors system in general to the joint motors. The optimal reference for this process is human motion, how great creator made the perfect sensors and delay free transmissions to transmit mind intended motions to specified joint. Referred to Figure 3, the inertias of the wearer leg and

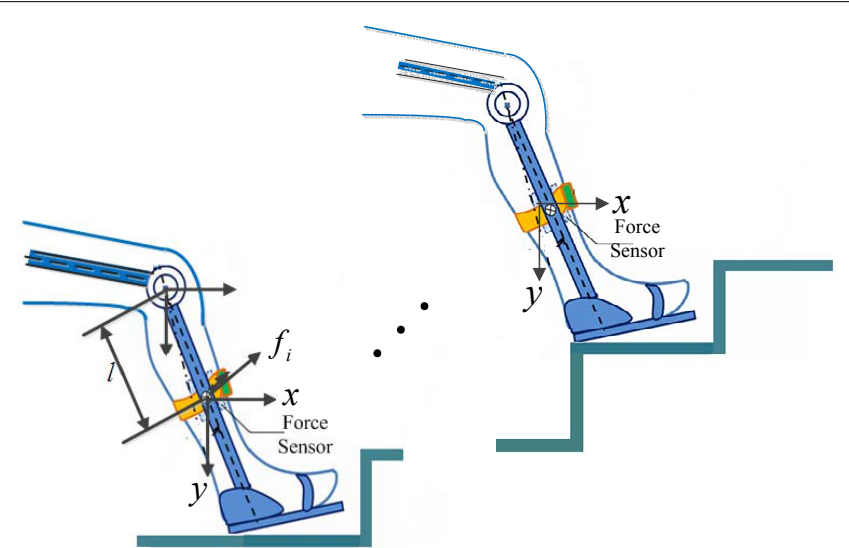

Figure 2: The schematic diagram of simple 1-DOF exoskeleton platform and resulting interaction force with the wearer when going upstairs. the exoskeleton are coupled by a damper $B_{c}$ and spring $K_{c}$ representing the coupled human and exoskeleton shank brace. The dynamic model of 1-DOF coupled human-exoskeleton system (shank with knee joint) can be represented as in Eqn. (4):

$$
J \ddot{\theta}(t)+B \dot{\theta}(t)+m g l \sin \theta(t)+C_{F} \operatorname{sign} \dot{\theta}(t)=\tau(t)+J^{T} f_{i}(t)
$$

where $J, B, m, l$ represent inertial moment, viscous friction coefficient, shank mass and length of the one DOF exoskeleton, respectively, $(\theta, \dot{\theta}, \ddot{\theta})$ represent the angle, angular velocity, and angular acceleration of the knee joint, $C_{F}$ represents Coulomb friction coefficient around knee joint, $(t)$ is actuation torque, $J^{T}$ is the jacobian transpose of the platform and $f_{i}(t)$ is the interaction force between human wearer and exoskeleton. We use stiffness $K$, mainly as a linearization of the gravitational torque acting on the shank, such that $\theta \approx K \theta[26]$. Consider the model of the coupled human-exoskeleton admittance control in Figures 3 and 4 we can rewrite Eqn. (4) as follows:

$$
J_{c} \ddot{\theta}(t)+B_{c} \dot{\theta}(t)+K_{c} \theta(t)+C_{F} \operatorname{sign} \dot{\theta}(t)=\tau(t)+J^{T} f i(t)
$$

The estimation of the dynamic model parameters in Eqn. (4) was defined for HUALEX walking speed control by T. H. Toan et al [22]. In this paper we define it for gait transitions control by ordinary admittance control method (OAC) we keep $C_{F}=1.724$ as estimated in [22]. To show the efficiency of our proposed variable admittance controller we demonstrate ordinary admittance controller first to evaluate ordinary performance. On the system navigation process the interaction force on shank contact is proportional to the wearer intended motion as follows:

$$
\Delta \theta(t)=J^{T} f i(t)
$$

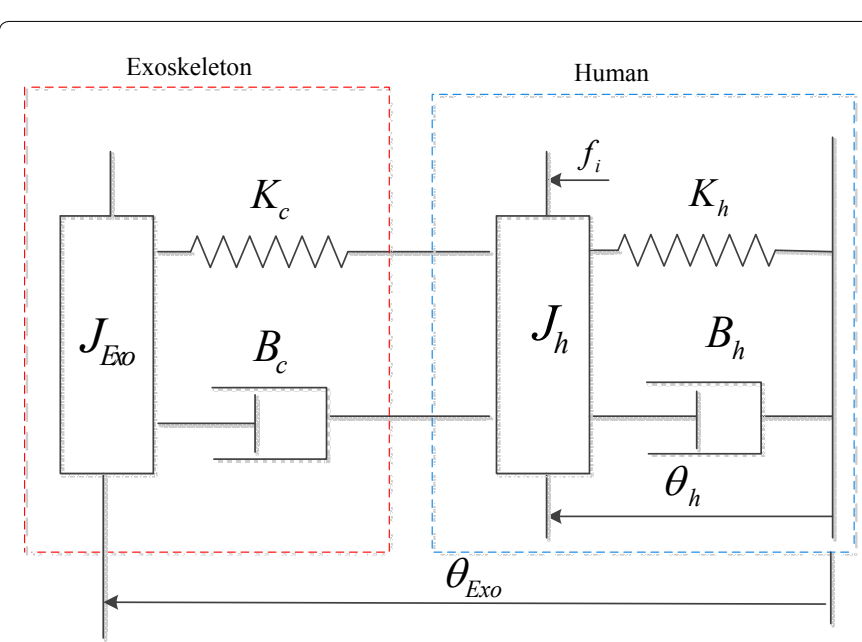

Figure 3: The ordinary admittance control model of the coupled humanexoskeleton system.

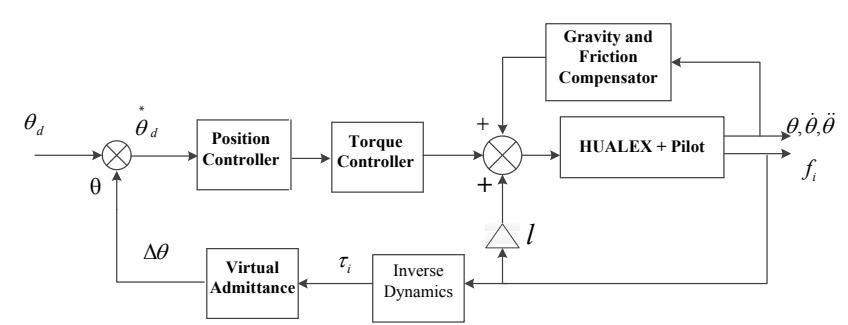

Figure 4: The main block diagram of admittance controller for coupled humanexoskeleton system interaction control. 
Therefore, if desired admittance parameters of the controller are invariant; the dynamic relationship between the exoskeleton and the human wearer through the admittance according to interaction force modeled as shown in Eqn. (6). According to the experimental simulation, the resulted tracking control of OAC also indicates performance illness in the gait transition situations. For defining $J$, BandK in Eqn.(6) define the vectors:

$$
\begin{aligned}
& \Phi(t)=[\ddot{\theta}(t) \dot{\theta}(t) \theta(t)]^{T} \\
& \Psi=\left[\begin{array}{lll}
J & B(t) & K
\end{array}\right]^{T}
\end{aligned}
$$

Assume relaxed wearer muscles operation i.e. $J^{T} f_{i}=0$, with the definitions in Eqn. (12) we can present Eqn. (4) in matrix form:

$$
\tau(t)=\Phi^{T}(t) \Psi
$$

Where, $\Phi^{T}$ is called the regression vector. The objective is to estimate the unknown parameter vector $\Psi$ from observations of $\tau(t)$ and the regression vector $\Phi(t)$. The best estimate of $\Psi$ can be obtained by minimizing the $L(t)$ least-square criterion which defined as

$$
L(t)=1 / 2\left[\tau(t)-\Phi^{T}(t) \hat{\Psi}\right]^{T}\left[\tau(t)-\Phi^{T}(t) \hat{\Psi}\right]
$$

The least-square method is the basic technique for parameters estimation [27]. The minimum of Eqn. (9) is our goal for parameter vector estimation:

$$
\frac{\partial \mathrm{L}(\mathrm{t})}{\partial \Psi}=0, \quad \Psi \rightarrow \hat{\Psi}
$$

We can say $\hat{\Psi}(t)$ is optimal if and only if following condition satisfied:

$$
\hat{\Psi}(t) \Phi T(t) \Phi(t)=\Phi^{T}(t) \tau(t)
$$

The condition in Eqn.(13) is called the normal equation. The online best estimation of parameter vector $\Psi$ based on recursive least-square (RLS) estimation can be achieved when assumed that the matrix $\Phi(t)$ has full rank [27]. Then the least-square estimate of $\hat{\Psi}(t)$ satisfies the recursive equations:

$$
\begin{aligned}
& \hat{\Psi}(t)=\hat{\Psi}(t-1)+K(t)\left[\tau(t)-\Phi^{T}(t) \hat{\Psi}(t-1)\right] \\
& K(\mathrm{t})=P(t-1) \Phi^{T}(t)\left[1+\Phi^{T}(t) P(t-1) \Phi(t)\right] \\
& P(t)=1-\left[K(t) \Phi^{T}(t)\right] P(t-1)
\end{aligned}
$$

From the experimental trails for flat terrain walking, stair ascent $170 \mathrm{~mm}$ height and $200 \mathrm{~mm}$ height the mean values for 10 trails are drawn in Table 1 . The performance of OAC demonstrated separately in different gait is pretty good on tracking the human wearer intentions. But the tracking error is a little bit unacceptable especially during transition time. In other words, OAC faces the problem of rapid change in physical interaction force when human intend to change gait which lead to the performance illness as shown in the Figure 5. The interaction force and OAC trajectory error for 2 cases of stairs height are shown.

\begin{tabular}{|l|c|c|c|}
\hline Parameter & $\begin{array}{c}\text { Flat terrain } \\
\text { walking }\end{array}$ & $\begin{array}{c}\text { Stair ascent } \\
\mathbf{( 1 7 0 ~ \mathbf { ~ m } )}\end{array}$ & $\begin{array}{c}\text { Stair ascent } \\
\mathbf{( 2 0 0} \mathbf{~ m m})\end{array}$ \\
\hline $\begin{array}{l}\text { Inertia } \\
J\left(\mathrm{Kgm}^{2}\right)\end{array}$ & 0.133 & 0.122 & 0.123 \\
\hline $\begin{array}{l}\text { Viscous friction } \\
\text { coefficient } \\
B(\mathrm{Nms} / \mathrm{rad})\end{array}$ & 6.282 & 7.371 & 7.446 \\
\hline $\begin{array}{l}\text { Stiffness parameter } \\
K(\mathrm{Nm} / \mathrm{rad})\end{array}$ & 33.921 & 31.120 & 29.414 \\
\hline
\end{tabular}

Table 1: The estimated parameters of lower shank and knee joint.

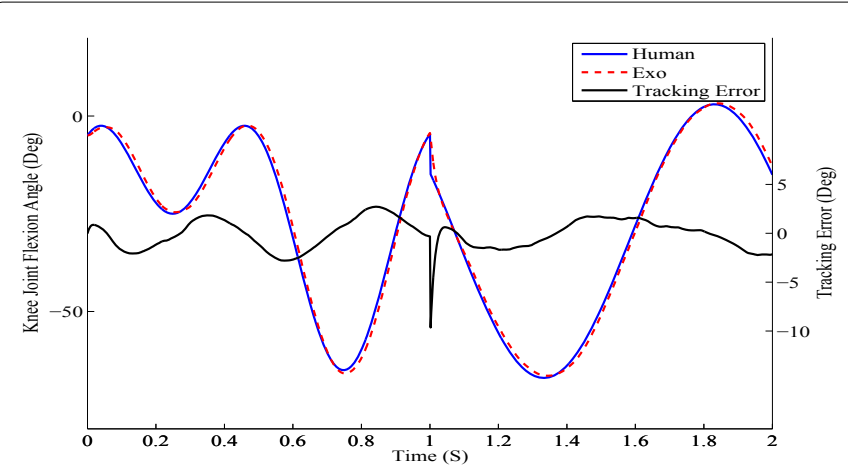

(a)

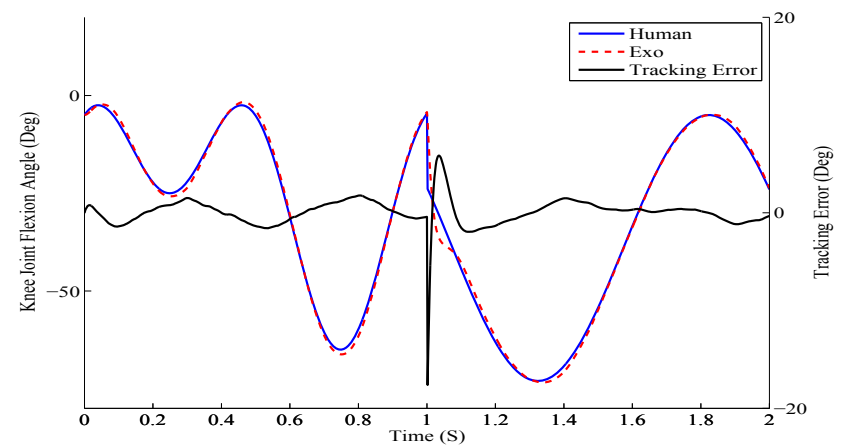

(b)

Figure 5: The performance of admittance control for coupled humanexoskeleton system gait transition 2 gait cycles (1 cycle flat terrain walking and 1 cycle stair ascent): (a) $170 \mathrm{~mm}$ height stairs; (b) $200 \mathrm{~mm}$ height stairs.

\section{Variable Admittance Control}

\section{A. Experimental simulations}

The modifications of OAC is needed due to ill effects on control process on the gait transition case, the overshoot and undershoots resulted on the measured interaction force must have special treatments for smooth transition. Our aim is to regulate admittance parameters to find an appropriate input command (trajectory reference change). It is evident that this input depends on wearer desired flexion angle and desired admittance parameters. To improve such OAC to pretty handle the uncertainties in dynamics model when human intend to change gait we introduce dynamic parameters estimation technique called variable admittance controller (VAC). The investigated VAC algorithm attempts to minimize the interaction force during whole navigation process of the coupled human-exoskeleton system, i.e. better tracking performance.

Force impulse and instantaneous end-effector oscillations are perfect system inputs to estimate VAC parameters. The Cartesian space intended position depends on the stair height in this case can be transformed to the joint space as the mean value of the difference between the desired and the actual trajectories. This difference $\Delta \theta(t)$ will be used to modify the input trajectory to get to the optimized trajectory:

$$
\theta_{h}^{*}(t)=\theta_{h}(t)+\Delta \theta(t)
$$

The block diagram of proposed variable admittance controller is shown in Figure 6 and the variable viscoelastic model of proposed VAC is drawn in Figure 7. 


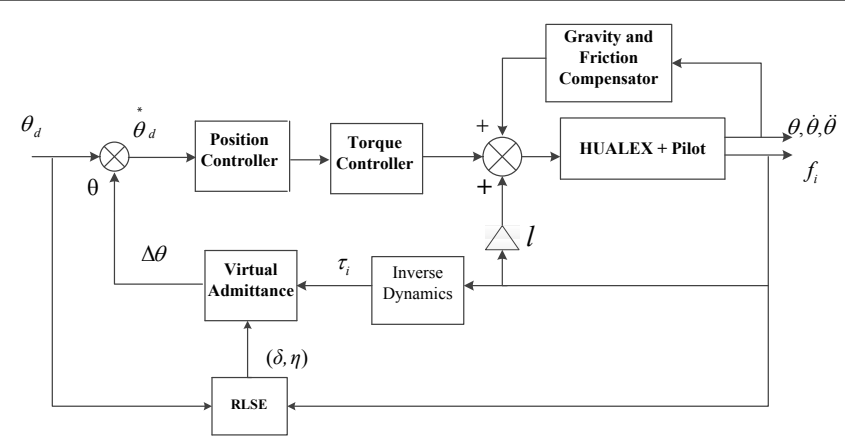

Figure 6: The proposed block diagram for variable admittance control scheme.

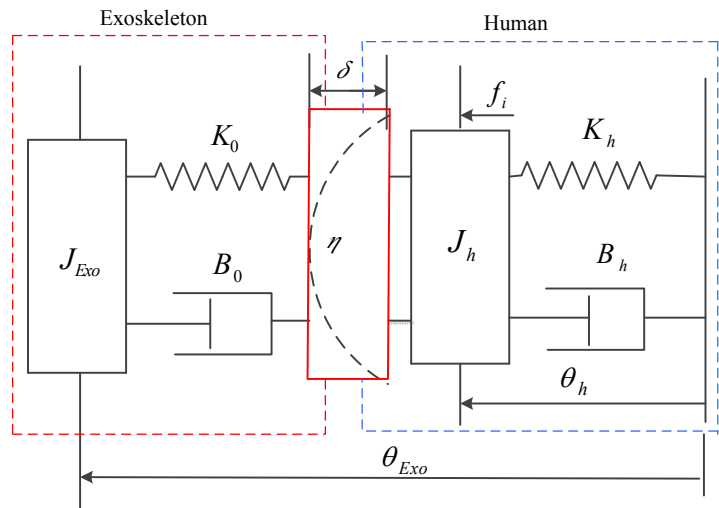

Figure 7: The variable visco-elastic model model of the variable admittance controller.

\section{B. Estimation of viscoelastic model parameters}

The dynamic process of input trajectory correction needs dynamic viscoelastic admittance parameters while keeping inertia constant:

$$
\begin{aligned}
& K_{d y n}=\delta K_{0} \\
& B_{d y n}=\eta B_{0}
\end{aligned}
$$

Where $K_{0}$ and $B_{0}$ represent the initial stiffness and viscous friction coefficient of OAC, depends on the current gait type as estimated in section 5 . The main goal of proposed VAC scheme is to achieve smooth trajectory tracking during and after gait transitions in other words is to minimize the tracking error when human intend to change gait type. We investigate a relation between VAC parameters and interaction force for accurate and perfect human trajectory tracking even when sudden change in current trajectory happens (gait transition). The dynamic stiffness and viscous are obtained on-line for smooth gait transitions. The linearity of the relation Eqn. (15) is experimentally proved for acceptable range of interaction force, this range is variable depends on human-exoskeleton system function.

$$
J_{C}\left(\ddot{\theta}_{h}(t)-\ddot{\theta}_{E x o}(t)\right)+B_{C}\left(\dot{\theta}_{h}(t)-\dot{\theta}_{E x o}(t)\right)+K_{C}\left(\theta_{h}(t)-\theta_{E x o}(t)\right)=J^{T}(t) f_{i}(t)
$$

Experimentally we estimate the dynamic visco-elastic model parameters from collected data applying recursive least square estimator (RLSE). The considered model for the estimation of dynamic visco-elastic parameters is:

$$
\eta B_{0} \Delta \dot{\theta}(t)+\delta K_{0} \Delta \theta(t)=\tau_{i}(t)
$$

The initial value of unknown parameters vector $\left[\begin{array}{ll}\delta K_{0} & \eta B_{0}\end{array}\right]^{T}$ are $\left[\begin{array}{ll}\delta & \eta\end{array}\right]^{T}=\left[\begin{array}{ll}1 & 1\end{array}\right]^{T}$, While $\left[\begin{array}{ll}K_{0} & B_{0}\end{array}\right]^{T}$ are variable through navigation process depend on the current gait. The collected samples are for 2 different gait cycle contains the transition. The inertia coefficient assumed constant. The changing rate of the interaction force between human wearer and exoskeleton are indicating the future changing of viscoelastic model parameters. The relations for efficient parameter estimation are investigated in this paper. The experimental results limits the values of $\delta$ and $\eta$ in the application on VAC for gait transition control, consider the variation of the stair height as follows:

$$
\begin{aligned}
& 0.0259 \leq \delta_{1} \leq 2.37750 .0248 \leq \delta_{2} \leq 2.3272 \\
& 0.1560 \leq \eta_{1} \leq 2.51970 .1549 \leq \eta_{2} \leq 2.5595
\end{aligned}
$$

where $\delta_{1}$ and $\eta_{1}$ are scaling coefficients of visco-elastic model for transition to $170 \mathrm{~mm}$ stairs, $\delta_{2}$ and $\eta_{2}$ for transition to $200 \mathrm{~mm}$. The method for adjusting admittance viscoelastic model parameters of the coupled human-exoskeleton system according to different gait types is promising towards fully autonomous human-exoskeleton system navigation. The on-line modification of input trajectory achieved successfully based VAC with dynamic viscoelastic parameters.

\section{Vac Performance Analysis}

The main advantage of VAC is the simple structure and implementation process, in other words has an availability for real applications and developing researches (low cost). VAC synchronizes the gait transition for human-exoskeleton system based on human intentions and trajectory construction, the performance is promising for further developments and applications. As shown in the simulation results VAC performs well and with more developing will be promising in Autonomous wearable Exoskeleton field. We take as inputs for control algorithm the pilot and exoskeleton joint angular positions, accelerations and interaction force between them. The stiffness and viscous friction parameters adjusted corresponding to the current gait type in order to keep the tracking error and interaction force as minimum as possible as in Eqn. (15). The proposed VAC scheme takes care of the transitions and allowing time for identification and adaptation to optimize performance. The performance of proposed control strategy for gait transitions is shown in Figure 8. The transition values of viscoelastic model parameters during transition for the different stairs height is shown in Table 2. The performance of proposed VAC algorithm is to determines the input needed to produce the desired plant performance (minimum tracking error). The comparison between OAC and VAC performance for gait transitions interaction force is shown in Figure 9, we compares the interaction force minimization and tracking error in $200 \mathrm{~mm}$ stairs case. The error correction appears big in $200 \mathrm{~mm}$ height stairs, as a result for the interaction force when human wearer intends to transit from flat terrain walking to stairs ascent.

The small disadvantages appear during proposed scheme simulations is the overshoots of interaction force, even though it was within the acceptable thresholds must have some treatments in future work. The transition process was achieved with high performance accuracy as depicted in Figure 8, with minimum tracking error and minimum interaction force.

\begin{tabular}{|c|c|c|}
\hline Parameter & $\mathbf{1 7 0} \mathbf{~ m m}$ Stairs height & $\mathbf{2 0 0} \mathbf{~ m m}$ Stairs height \\
\hline Ktr. & 10.238 & 10.535 \\
\hline Btr. & 16.353 & 14.647 \\
\hline
\end{tabular}

Table 2: The transition values of visco-elastic model parameters. 


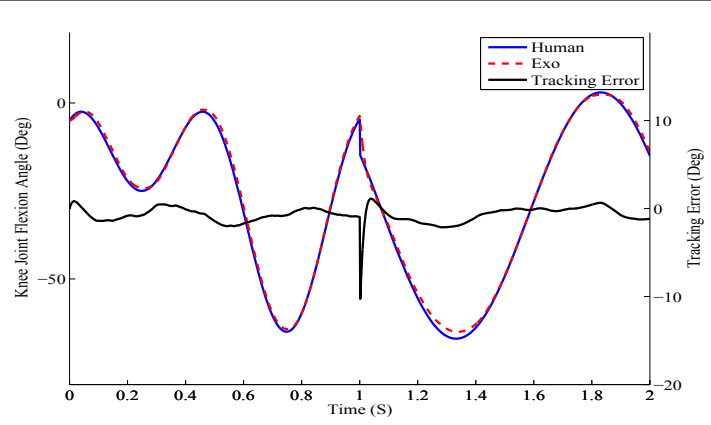

(a)

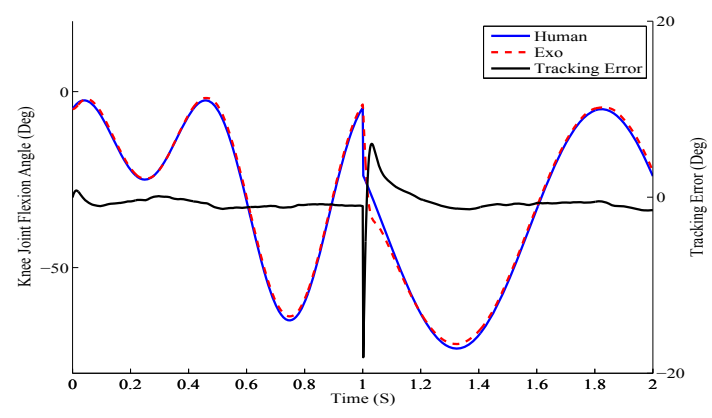

(b)

Figure 8: The performance of VAC for coupled human- exoskeleton system gait transition 2 gait cycles (1 cycle flat terrain walking and 1 cycle stair ascent): (a) $170 \mathrm{~mm}$ height stairs; (b) $200 \mathrm{~mm}$ height stairs.

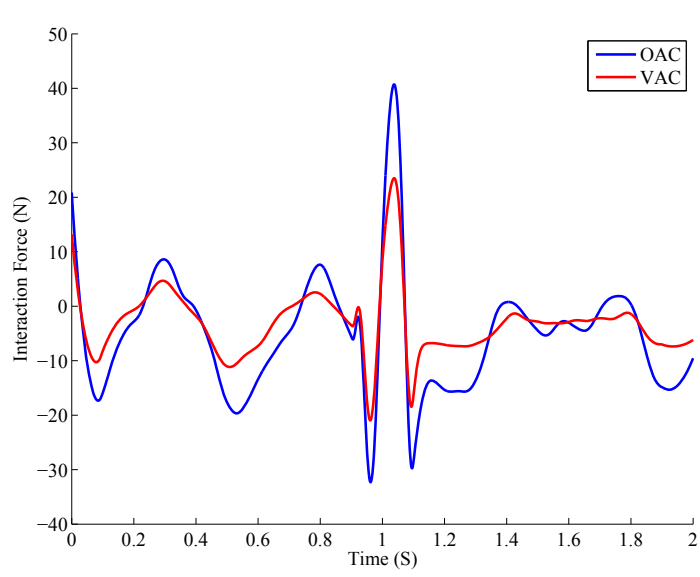

Figure 9: The proposed VAC strategy interaction force compared to OAC.

\section{Conclusions and Future Works}

Our control method synchronizes the gait transition for humanexoskeleton system based on human intentions and trajectory construction, the performance is promising for further developments and applications. The proposed VAC scheme for online gait transition control system with limited gait modes (flat terrain walking and stairs ascent) will find a more attentions in future developments forwards fully autonomous HUALEX robotic system. As shown in results our technique performs well and more developing will be promising work in Autonomous wearable Exoskeleton field. In future work will focus on the system response optimization to minimize the transition error, then develop our algorithm to work in obstacle avoidance in general and adapt to difference stairs height and tread length this will overcome the proposed technique limitations, then will go forward for practical implementation in our lab Exoskeleton. The interaction force convex (overshoots or undershoots) will find some attention in our future work to be minimized.

\section{Acknowledgments}

This work was support by NSFC (No. 61503060, 6157021026), Fundamental Research Funds for the Central Universities (ZYGX2014Z009) and SRF for ROCS, SEM.

\section{References}

1. Kazerooni H, Racine JL, Huang L, Steger R (2005) On the control of the berkeley lower extremity exoskeleton (BLEEX). International Conference of Robotics and Automation pp: 4353-4360.

2. Riener R, Rabuffetti M, Frigo C (2002) Stair ascent and descent at different inclinations. Gait and Posture 15: 32-44.

3. Hoover CD, Fulk GD, Fite KB (2013) Stair ascent with a powered trans femora prosthesis under direct myoelectric control. IEEE/ASME Transaction on Mechatronics 18: 1191-1200.

4. Amirudina AN, Parasuramanb S, AhmedKhanc AK, Elamvazuthid I (2014) Biomechanics of hip, knee and ankle joint loading during ascent and descent walking. International Conference on Medical and Rehabilitation Robotics and Instrumentation pp: 336-344.

5. Andriacchi TP, Galante JO, Fermier RW (1982) The influence of total kneereplacement design on walking and stair climbing. The Journal of Bone and Joint Surgery 64: 1328-1335.

6. Bergmann G, Graichen F, Rohlmann A (1995) Is staircase walking a risk for the fixation of hip implants?. Journal of Biomechanics 28: 535-553.

7. Amor HB, Neumann G, Kamthe S, Kroemer O, Peters J (2014) Interaction primitives for human-robot cooperation tasks. IEEE International Conference on Robotics and Automation pp: 2831-2837.

8. Ikemoto S, Amor HB, Minato T, Jung B, Ishiguro H (2012) Physical human-robot interaction: mutual learning and adaptation. IEEE Robotics and Automation Magazine 19: 24-35.

9. De Rossi SMM, Lenzi T, Vitiello N, Donati M, Persichetti A, et al. (2011) Development of an in-shoe pressure-sensitive device for gait analysis. Engineering in Medicine and Biology Society, EMBC, Annual International Conference of the IEEE pp: 5637-5640.

10. Liu T, Inoue Y, Shibata K (2010) A wearable ground reaction force senso system and its application to the measurement of extrinsic gait variability Sensors 10: 10240-10255.

11. Hassan M, Kadone H, Suzuki K, Sankai Y (2014) Wearable Gait Measurement System with an Instrumented Cane for Exoskeleton Control. Sensors 11: 17051722.

12. Crea S, Donati M, De Rossi SMM, Oddo CM, Vitiello N (2014) A wireless flexible sensorized insole for gait analysis. Sensors 14: 1073-1093.

13. Tonietti G, Schiavi R, Bicchi A (2005) Design and Control of a Variable Stiffness Actuator for Safe and Fast Physical Human-Robot Interaction. International Conference on Robotics and Automation pp: 526-531.

14. Hogan N (1984) Impedance Control: An Approach to Manipulation. American Control Conference, pp: 304-313.

15. Miller LM, Rosen J (2010) Comparison of multi-sensor admittance control in join space and task space for a seven degree of freedom upper limb exoskeleton. Proceedings of the 3rd IEEE RAS and EMBS International Conference on Biomedical Robotics and Biomechatronics, pp: 70-75.

16. Okunev V, Nierhoff T, Hirche S (2012) Human-preference-based contro design: adaptive robot admittance control for physical human-robot interaction. The 21st IEEE International Symxposium on Robot and Human Interactive Communication pp: 443-448.

17. Carmichael MG, Liu D (2013) Admittance control scheme for implementing model-based assistance-as-needed on a robot. 35th Annual International Conference of the IEEE EMBS pp: 870-873.

18. Lee BK, Lee HD, Lee JY, Shin K, Han JS, et al. (2012) Development of dynamic model-based controller for upper limb exoskeleton robot. 2012 IEEE International Conference on Robotics and Automation pp: 3173-3178. 
Citation: Ahmed AIA, Cheng H, Lin X, Omer M, Atieno JM (2016) Variable Admittance Control for Climbing Stairs in Human-Powered Exoskeleton Systems. Adv Robot Autom 5: 157. doi: 10.4172/2168-9695.1000157

19. Yu W, Rosen J, Li X (2011) PID Admittance control for an upper limb exoskeleton. American Control Conference pp: 1124-1129.

20. Augugliaro F, Andrea RDS (2013) Admittance control for physical humanquadrocopter interaction. European Control Conference pp: 1805-1810.

21. Oda M, Zhu C, Suzuki M, Luo X, Watanabe H, et al. (2010) Admittance based control of wheelchair typed omnidirectional robot for walking support and power assistance. 19th IEEE International Symposium on Robot and Human Interactive Communication pp: 159-164.

22. Tran HT, Cheng H, Duong MK, Zheng H (2014) Fuuzy-based Impedance Regulation for Control of the Coupled Human-Exoskeleton System. IEEE International Conference on Robotics and Biomimetics pp: 986-992.

23. Tran HT, Cheng H, Lin X, Huang R (2014) The relationship between physical human-exoskeleton interaction and dynamic factors: using a learning approach for control applications. Science China Information Science 57: 1-13.
24. huang $\mathrm{R}$, Cheng $\mathrm{H}$, Chen $\mathrm{Q}$, Tran $\mathrm{Ht}$, Lin $\mathrm{X}$ (2015) Interactive Learning For Sensitivity Factors Of A Human-Powered Augmentation Lower Exoskeleton. IEEE/RSJ International Conference on Intelligent Robots and Systems, pp: 6409-6415.

25. Ka M, Cheng H, Toan TH, Jing Q (2015) Minimizing human-exoskeleton interaction force using compensation for dynamic un-certainty error with adaptive rbf network. Journal of Intelligent and Robotic Systems, pp: 1-21.

26. Colgate JE, Ollinger GA, Peshkin MA, Goswami A (2007) A 1-DOF assistive exoskeleton with virtual negative damping: effects on the kinematic response of the lower limbs. IEEE/RSJ International Conference on Intelligent Robots and Systems, pp: 1938-1944.

27. Astrom KJ, Wittenmark B (1995) Adaptive control, (2ndedn) Addison Wesley, Reading. 